\title{
Hypokalaemia in Sjögren's syndrome: the missing piece
}

\author{
Authors: Umer Farooq Khan, ${ }^{A}$ Lee Chun Tsu, ${ }^{B}$ Kong-Bing Tan, ${ }^{C}$ Jingxiang Huang ${ }^{D}$ and Audrey LiAnn Wong ${ }^{\mathrm{E}}$
}

\begin{abstract}
A 58-year-old Chinese woman with well controlled type 1 diabetes mellitus presented with acute and progressive bilateral lower limb weakness. Investigations revealed severe hypokalaemia $(1.3 \mathrm{mmol} / \mathrm{L})$ and hypophosphataemia ( $<0.32 \mathrm{mmol} / \mathrm{L}$ ) with rhabdomyolysis and electrocardiogram changes, without other concurrent biochemical abnormalities. Immediate intravenous and oral potassium and phosphate replacement was initiated with objective improvement in weakness with replenished electrolyte levels. Urine studies confirmed renal potassium wasting. Further history revealed frequent dental caries, xerostomia and recent weight loss. A computerised tomography scan showed atrophy of her salivary glands and a skin lesion biopsied by her GP in the past had been histologically characterised as anetoderma. The constellation of these findings and subsequent positive anti-SSA/SSB levels confirmed her diagnosis of primary Sjögren's syndrome (PSS). PSS has a wide spectrum of renal involvement and should be a differential diagnosis when investigating interstitial nephritis and electrolyte abnormalities, particularly in patients with coexisting autoimmune conditions.
\end{abstract}

KEYWORDS: Anetoderma, hypokalaemia, Sjögren's syndrome

\section{Case presentation}

A 58-year-old woman presented to the emergency department (ED) with a 1-day history of rapidly progressive weakness of bilateral lower limbs. She had no back pain, leg numbness, urinary or bowel symptoms. She was otherwise well, afebrile with stable vital signs. On examination, she had poor dentition with multiple caries. There was grade 3 (Medical Research Council scale for muscle power) proximal muscle weakness in shoulder abduction and hip flexion with preserved power of the distal muscle groups. There was no fatigability and

Authors: ${ }^{A}$ resident in internal medicine, National University Health System, Singapore; ${ }^{B}$ senior resident in haematology, National University Health System, Singapore and National University Cancer Institute Singapore, Singapore; ' professor of pathology, National University Health System, Singapore; ${ }^{D}$ associate consultant in pathology, National University Health System, Singapore; Eassociate professor of internal medicine, National University Health System, Singapore and Yong Loo Lin School of Medicine, National University Singapore, Singapore

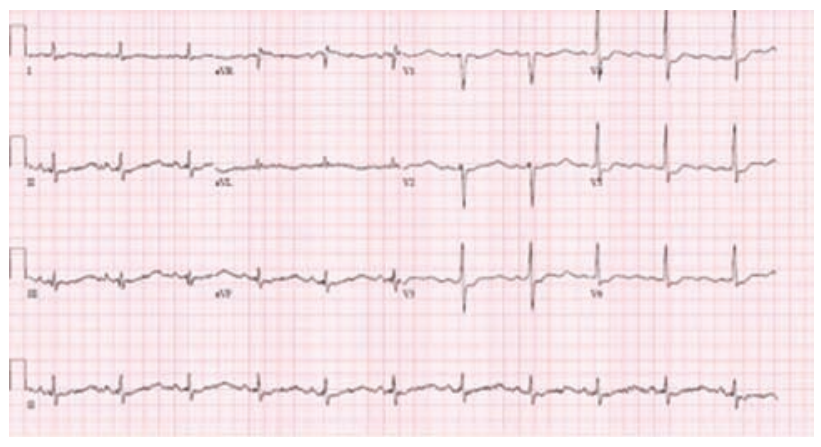

Fig 1. Electrocardiogram of patient showing $T$ wave flattening, $U$ waves, ST depression and QTc prolongation.

deep tendon reflexes in all four limbs were hyporeflexic. The rest of the examination was unremarkable. Her comorbidities included type 1 diabetes mellitus, which was diagnosed at the age of 40 . Her HbAlc was $7.5 \%$ without micro- or macrovascular complications. She reported having undergone a minor surgical procedure for a skin papule many years previously.

Initial investigations revealed severe hypokalaemia of $1.3 \mathrm{mmol} / \mathrm{L}$ (normal range $3.5-5.0 \mathrm{mmol} / \mathrm{L}$ ), and hypophosphatemia of $<0.32 \mathrm{mmol} / \mathrm{L}$ (normal range 0.85 $1.45 \mathrm{mmol} / \mathrm{L}$ ). Serum bicarbonate, magnesium, adjusted calcium, pH of blood gas, full blood count and thyroid function were normal. There was evidence of rhabdomyolysis as creatinine kinase was 2,508 U/L (normal range 20-300 U/L). There was associated QTc prolongation to $467 \mathrm{~ms}$, U waves and other hypokalaemic electrocardiogram changes (Fig 1). Review of her previous laboratory results showed normokalaemia.

\section{Diagnosis}

The exact cause of the patient's clinical findings was not immediately apparent. As there was no evidence of obvious renal and gastrointestinal losses, other causes of transcellular shift of hypokalaemia were considered; for example, renal tubular acidosis (RTA) and thyrotoxic periodic paralysis, but were deemed to be unlikely because of the lack of metabolic acidosis and normal thyroid-stimulating hormone and thyroxine. Eventually, hypokalaemic periodic paralysis was thought to be the most likely provisional diagnosis after consulting with neurology, but her long and short exercise tests on nerve conduction studies were negative. 


\section{Initial management}

In the $\mathrm{ED}$, the patient received $40 \mathrm{mmol}$ of oral potassium chloride ( $\mathrm{KCl}$ ) and intravenous (IV) $\mathrm{KCl}$ was started at $10 \mathrm{mmol} /$ hour. She was admitted to the telemetry unit for close cardiac monitoring while she received aggressive IV $\mathrm{KCl}$ replacement at $10 \mathrm{mmol} /$ hour together with $2.5 \mathrm{mmol} /$ hour of IV potassium dihydrogen phosphate. She also received oral $\mathrm{KCl}$ at $20 \mathrm{mmol}$ 4-hourly. There was marked improvement in the lower limb power clinically from 3 to 4 by the second day of admission when the potassium was $2.8 \mathrm{mmol} / \mathrm{L}$. By the fourth day of admission, she had received a total of $230 \mathrm{mmol}$ of potassium and $20 \mathrm{mmol}$ of phosphate and her potassium and phosphate normalised to $3.6 \mathrm{mmol} / \mathrm{L}$ and $0.89 \mathrm{mmol} / \mathrm{L}$, respectively. Electrocardiogram abnormalities and creatine kinase levels normalised as potassium levels improved.

\section{Case progression}

Further investigations confirmed that the hypokalaemia was due to urinary losses as 24-hour urine collection showed urinary potassium of $81 \mathrm{mmol} /$ day. There was no evidence to suggest acid-base abnormalities in relation to renal tubular acidosis; renin and aldosterone levels and ratio were within normal limits. There were no urinary sediments or proteinuria to suggest glomerulonephritis. On further questioning, the patient reported unintentional weight loss of $6 \mathrm{~kg}$ over the previous 2 months, which she attributed to a lack of appetite. She claimed that food did not taste as good as before. She had also frequently visited her dentist for dental caries and was told she had a dry mouth.

As part of a malignancy workup for weight loss, a computerised tomography scan of her neck, thorax, abdomen and pelvis was done; it showed no significant findings except atrophy of her salivary glands. With this peculiar clinical development, our impression of her overall clinical findings of severe renal potassium wasting, xerostomia and salivary gland atrophy was that of primary Sjögren's syndrome (PSS). Her GP was contacted, the histological report of the skin papule excised in the past was evident for anetoderma (Fig 2). Anetoderma is characterised by flaccid-appearing skin lesions that show absence of dermal elastic fibres on biopsy. The condition may be primary or secondary and is associated with a variety of systemic diseases, including autoimmune diseases such as PSS. ${ }^{1}$

An autoimmune workup showed polyclonal IgG elevation, with anti-SSA/Ro values of 4.1 (positive) and anti-SSB/La of $>5.0$ (strongly positive). However, her complement 3 was $81 \mathrm{mg} / \mathrm{dL}$ (normal range 85-185) and complement 4 was $30 \mathrm{mg} / \mathrm{dL}$ (normal range $10-50$ ), with a negative anti-dsDNA. Antinuclear antibody titres were 1:640, but other autoimmune markers, such as anti-RNP, anti-Sm, anti-Jo1 and anti-Scl70, were negative.

The patient was discharged with oral $1.8 \mathrm{mmol}$ potassium per day, with outpatient follow-up with rheumatology for potassium level monitoring and long-term management of Sjögren's syndrome.

\section{Discussion}

Hypokalaemia is a common finding in laboratory testing of patients in the ED and severe hypokalaemia $(\mathrm{K}<2.9 \mathrm{mmol} / \mathrm{L})$ is

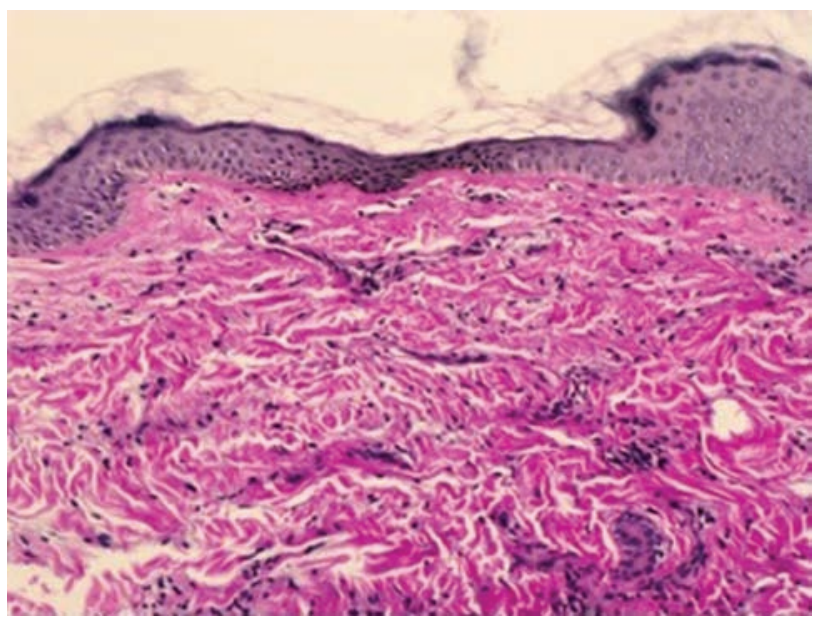

Fig 2. Photomicrograph of the skin biopsy shows paucity of elastic fibres in the dermis, compatible with anetoderma. Elastic-Van Gleson stain, original magnification $\times 100$.

associated with increased mortality with adjusted hazard ratio of $2.17 .^{2}$ A decrease in potassium concentration from 4.0 to $3.0 \mathrm{mmol} / \mathrm{L}$ corresponds to a potassium deficit of $350 \mathrm{mmol} .^{3} \mathrm{In}$ patients with cardiac and neuromuscular involvement, rapid IV and oral replacement is indicated, with serial serum potassium checks every 6-8 hours to guide replacement strategy. The rate of administration of potassium should not exceed $>60 \mathrm{mmol} /$ hour because of the risk of arrhythmias. ${ }^{3}$ Infusions should not contain dextrose or bicarbonate as these solutes may increase intracellular shift of potassium.

Sometimes, the causes of hypokalaemia can be occult and challenging to diagnose. PSS with renal involvement is one of them. The reported prevalence of renal involvement in PSS varies across studies because of the different definitions used for renal involvement. The spectrum of renal involvement in PSS consists of distal or proximal RTA, glomerulonephritis, and acute or chronic tubulointerstitial nephritis, ${ }^{4}$ which is also very commonly seen in lupus. ${ }^{5}$ Most of the hypokalaemia reported in PSS is due to distal RTA. ${ }^{4}$ In this case, severe hypokalaemia occurred without acidosis, which is a rare and seldom reported entity. Wrong et al ${ }^{6}$ reported a case series of patients with immune-mediated potassium wasting interstitial nephritis in a variety of autoimmune diseases, including PSS. The pathogenesis of hypokalaemia in this condition is explained by potassium wasting due to renin and aldosterone hypersecretion secondary to renal sodium loss. Urinary acidification is usually intact or minimally disturbed; hence, there is an absence of metabolic acidosis. ${ }^{6}$

\section{Conclusion}

In this scenario, connecting the dots is challenging without understanding the complexity of tubular function and clinical pathology of PSS. Nonetheless, one should suspect autoimmune conditions in patients with type 1 diabetes mellitus as autoimmune diseases have the tendency to coexist. The skin nodule, which turned out to be anetoderma (Fig 2), was the missing piece that completed the whole clinical picture. ${ }^{1}$ 


\section{Key learning points}

> Profound hypokalaemia can be life threatening and patients should be monitored closely while aggressive intravenous replacement is initiated.

$>$ Autoimmune conditions tend to coexist and should be considered as differential diagnoses in patients with pre-existing conditions such as type 1 diabetes. Because of multisystem involvement of autoimmune conditions, thorough investigation is often required to arrive at a diagnosis.

$>$ Sjögren's syndrome can have a spectrum of renal manifestations, most commonly distal renal tubular acidosis or tubulointerstitial nephritis.

\section{Conflicts of interest}

The authors have no conflicts of interest to declare.

\section{Acknowledgements}

Consent was obtained from the patient to publish the clinical details and images in this article.

\section{References}

1 Herrero-González JE, Herrero-Mateu C. Primary anetoderma associated with primary Sjögren's syndrome. Lupus 2002;11:124-6.

2 Jensen HK, Brabrand M, Vinholt PJ, Hallas J, Lassen AT. Hypokalemia in acute medical patients: risk factors and prognosis. Am J Med 2015;128:60-7.e1.

3 Lin SH. A practical and pathophysiologic approach to hypokalemia. Hong Kong J Nephrol 2008;10:14-26.

4 Pertovaara M, Korpela M, Kouri T, Pasternack A. The occurrence of renal involvement in primary Sjogren's syndrome: a study of 78 patients. Rheumatology 1999;38:1113-20.

5 Cameron JS. Lupus nephritis. J Am Soc Nephrol 1999;10:413-24.

6 Wrong OM, Feest TG, Maciver AG. Immune-related potassiumlosing interstitial nephritis: a comparison with distal renal tubular acidosis. Q J Med 1993;86:513-42.

Address for correspondence: Dr U F Khan, 1 E Kent Ridge Road, Level 10, 119228 Singapore.

Email: umer_khan@nuhs.edu.sg 